\title{
A New Architecture for Group Replication in Data Grid
}

\author{
Leila Azari \\ Department of Computer, \\ Science and Research Branch, \\ Islamic Azad University, \\ Khouzestan, Iran
}

\author{
Mashalla Abbasi Dezfouli \\ Department of Computer, \\ Science and Research Branch, \\ Islamic Azad University, \\ Khouzestan, Iran
}

\begin{abstract}
Nowadays, grid systems are vital technology for programs running with high performance and problems solving with largescale in scientific, engineering and business. In grid systems, heterogeneous computational resources and data should be shared between independent organizations that are scatter geographically. A data grid is a kind of grid types that make relations computational and storage resources. Data replication is an efficient way in data grid to obtain high performance and high availability by saving numerous replicas in different locations e.g. grid sites. In this research, we propose a new architecture for dynamic Group data replication. In our architecture, we added two components to OptorSim architecture: Group Replication Management component (GRM) and Management of Popular Files Group component (MPFG). OptorSim developed by European Data Grid projects for evaluate replication algorithm. By using this architecture, popular files group will be replicated in grid sites at the end of each predefined time interval.
\end{abstract}

Keywords: data Grid; data replication; group replication; architecture; replica.

\section{INTRODUCTION}

In grid systems, heterogeneous computational resources and data should be shared between independent organizations that are scatter geographically [1]. On the other hand, the data Grid is a kind of grid services that provides services and infrastructure for distributed data applications with large volumes of data. These basic services which are provided by the data grid architecture are: storage systems, data access and meta-data services [2]. Data replication is one of the main services to manage volume data in data grid. This service save replica in different locations. Therefore, when each user needs those files, it will be available locally and causes reducing access latency time, response time and bandwidth consumption. So far various strategies have been presented in this field. Grid architecture has an efficient factor in replication technique. There are different architectures for data grid that all of them are based on hierarchal data model [3]. Data grids can be classified into two types [4]: multi-tier data grids that first introduced by the MONARC project [5], and cluster data grids that initially proposed by Chang et al. [6]. Multi-tier data grid architecture contains two models, tree model and graph (peer to peer) model. In tree model, Each node can communicate only to the parent node and there is only one path from a leaf to the root. Because of architecture failure we can not simply add nodes to the grid. For example, at GriPhyN project has been used tree model. There are five tiers in this model. In this multi-tier data grid architecture, the root site holds all files that are produced initially in the data grid. Next tiers are national center, regional center, work groups and leafs node represents desktop. In graph model, grid sites connect to each others as a peer to peer architecture. In this model, some of the limitations of the tree type are improved. In 2001, six replication techniques were presented by Ranganathan and foster [7] for a multi-tier data grid. In 2002 Ranganathan [8] presented a replication technique based on peer to peer communities. A cluster represents an organization unit which is a group of sites that are geographically close to each others. A cluster data grid consists of $\mathrm{n}$ clusters connected by the Internet [9]. Recent researchers have used hierarchal models as a basis and proposed other architectures for replication techniques. The most above mentioned techniques are the based on a single replication. If a replica does not exist locally in a requester grid site replication techniques will replicate it to that grid site. In this paper, we propose a new architecture for dynamic group data replication which has two added components to OptorSim architecture: Group Replication Management component (GRM) and Management of Popular Files Group component (MPFG). This paper is organized in the following way: Section 2 gives a brief introduction of related work on architecture for data grid. In section 3, the architecture of OptorSim is presented. Section 4 describes proposed architecture and finally concludes our presents some future work.

\section{RELATED WORK}

Chang et al. [6] presented a hierarchical architecture with concept of clustering. Cluster grid is a simple hierarchical form of a grid system. There are two kinds of communications between grid sites in a cluster grid. Intra-communication is the communication between grid sites within the same cluster and inter-communication is the communication between grid sites across clusters. Network bandwidth between grid sites within a cluster will be larger than across clusters. In this architecture, clusters are connected via internet. A job scheduling policy called HCS is presented based on this architecture that considers not only computational capability and data location but also cluster information. Also a dynamic replica optimization strategy (HRS) is suggested where the nearby data has a higher priority to access than to generate new replicas. The simulation results showed that HCS successfully reduced data access time and the amount of intercluster-communications in comparison with LRU (Least Recently Used), LFU (Least Frequently Used), BHR (Bandwidth Hierarchy-based Replication) [10]. Since the file transmission time is the most important factor to influence the job execution time for data-intensive jobs in data grids, HCS with HRS can reduce the file transmission time effectively by virtue of valid scheduling and proper data replication. Sepahvand et al. [11] presented three-level hierarchical architecture. This architecture represents a real structure in most academic data centers, cities and countries. First level is grid sites that contain personal computers. These sites are connected by a high bandwidth network. Second level contains LAN. Compared with first level, LANs are connected by a low bandwidth network. Finally, there are 
several LANs in each region which are connected via Internet having a low bandwidth network. If there is not space for replica, only those file will be deleted that have a low cost of transfer i.e. considering the bandwidth between source and destination. So it deletes those files that are available in local LAN. In comparison to BHR algorithm which considers 2level, the 3 level has performed better and it is more realistic. The simulation results with OptorSim have showed better performance comparing to LRU and BHR.

Sashi and Thanamani [12] presented a replication algorithm which is called Modified BHR with multi-tier architecture based on region. Region comprise group of sites that are geographically located close together. Each region has a header. All files were produced in a master site and were distributed in each region headers. The Modified BHR Region increases the data availability by replicating files within the region to the region header and storing them in the site where the file has been accessed frequently. Instead of storing files in many sites, Modified BHR stores them in a particular site so that the storage usage can be reduced. In comparison to No Replication, (LFU), (LRU) and BHR algorithms, Modified BHR reduces mean job execution time and the network traffic too.

Chang et al. [9] presented a replication algorithm which is called Latest Access Largest Weight (LALW) with multi-tier architecture based on a centralized data replication management which has a Dynamic Replication Policymaker (Policymaker) that responsible for replica management. This architecture contains grid sites within a cluster. There is a header used to maintain the site's information in a cluster. In the time interval, Policymaker sends a request for collecting site's information. Each header sends the information of accessed files from all sites to Policymaker. LALW selects a popular file for replication and calculates a suitable number of copies and grid sites for replication. According to access frequencies for all files that have been requested, a popular file is found and replicated to grid sites. In LALW, the data access records in the nearer past have higher weigh and higher value of references. The simulation results showed that the average job execution time of LALW is similar to LFU optimizer, but exceeded in terms of effective network usage. Saadat and rahmani [13] presented a replication algorithm named PDDRA hierarchical architecture supporting their dynamic replication technique. Grid sites is first level several grid sites constitute a virtual organization (VO). Next level is Local Server for each VO. Regional Server (RS) comprise the final level. Each RS consists of one or more VOs. Due to the far distance between the RS, they are connected via internet which has low bandwidth. Sites within a VO have similar interests. PDDRA predicts future needs of grid sites and prefetches a sequence of files to the requester grid site, so the next time that this site needs a file, it will be locally available. The simulation results showed that PDDRA has better performance in comparison with No Replication, LRU, LFU, EcoModel, EcoModel Zipf-like distribution and PRA [14] in terms of job execution time, effective network usage, total number of replications, hit ratio and percentage of storage filled.

\section{THE ARCHITECTURE OF OPTORSIM}

OptorSim is used as the simulator tool written in Java to evaluate the performance of replication strategies. It was developed by the European Data Grid projects [15]. It was developed to study the effectiveness of replica optimization algorithms within a data grid environment [16]. The architecture used in OptorSim is the CMS testbed architecture [17]. Figure 1, describes the Grid topology; that is, the resource available and the network connections to other sites. Sites are represented as green nodes and routers as red nodes. In this architecture, there are twenty sites, two of which only have a storage element and act as the master node. CERN and FNAL are considered the master sites where data is produced initially. The master site has the most capacity, which allows it to hold all the master files at the beginning of the simulation. The storage capacity of the master site is $100 \mathrm{~GB}$, and the storage capacity of all other sites is $50 \mathrm{~GB}$. Each data file to be accessed is 1 GB. Jobs are processed in the remaining sites that have computing elements. There are eight routers that are used to forward requests to other sites.

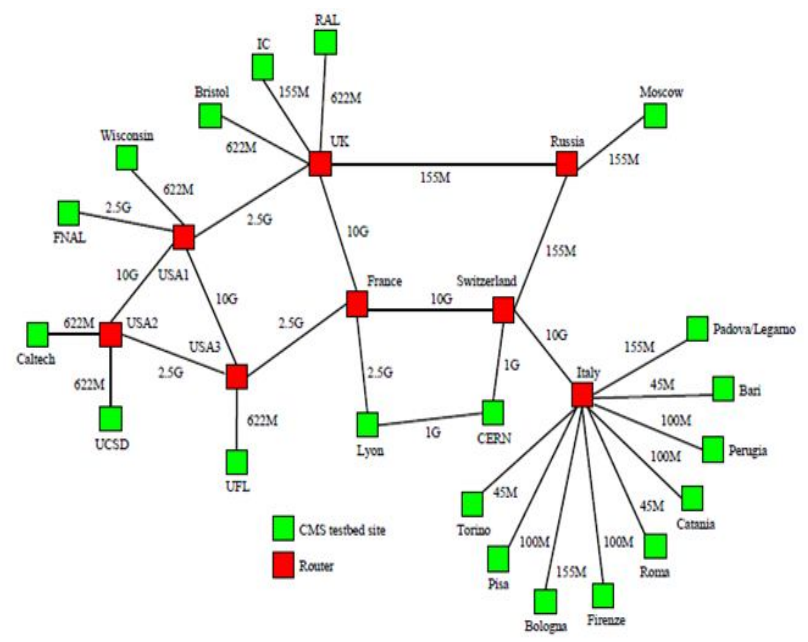

Figure 1. CMS topology

The internal architecture [18] of OptorSim and the content of each site is illustrated in Figure 2, Each site may provide computational and data storage resources called the Computing Element (CE) and the Storage Element (SE). CEs run jobs by processing data files, which are stored in the SEs. A Resource Broker (RB) controls the scheduling of jobs to Grid Sites, and schedules jobs to CEs according to scheduling algorithm. Each site handles its file content with Replica Manager (RM), within which a Replica Optimizer (RO) contains the replication algorithm which drives automatic creation and deletion of replicas [18]. Jobs are submitted to the grid over a period of time via the RB. The RB schedules each job to the CE with the goal to improve the overall throughput of the grid. RM at each site manages the data flow between sites. The RO inside the RM is responsible for the selection and dynamic creation and deletion of file replicas.

\section{PROPOSED ARCHITECTURE}

Our proposed architecture contains Local Servers (LS) and grid sites. We have used virtual organization concept in our 


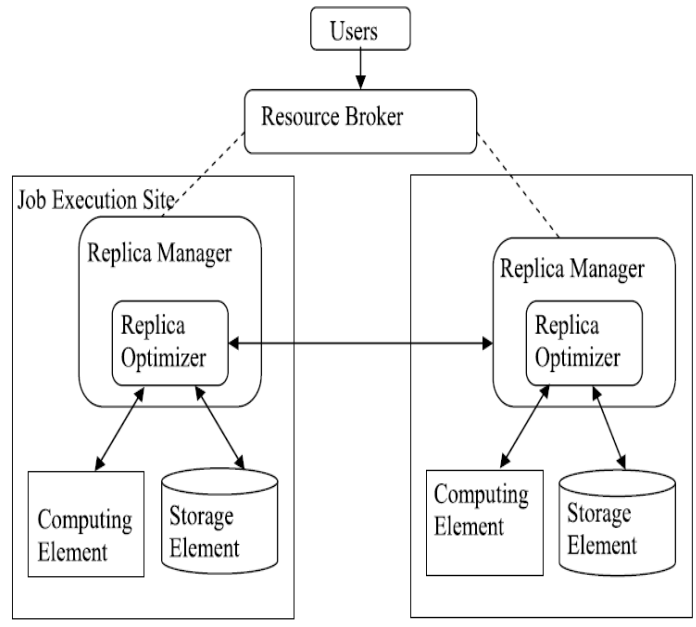

Figure 2. OptorSim architecture

architecture. Collection of grid sites constitute a VO. Grid sites within a VO have similar interests and request similar files. There is also a LS within each VO. LSs are connected via internet which has low bandwidth. Therefore, speed of data access within VO is larger than across VOs. OptorSim [19] assumes that each grid sites contains zero or more Computing Elements (CE) to run jobs and zero or more Storage Elements (SE) to store files, or a combination of both. In OptorSim, there is a Resource Broker (RB) that controls job scheduling between different CEs. Figure 3 shows the proposed architecture. First of all, end users submit their jobs to RB. Then RB schedules jobs between LS by scheduling optimization and are appropriate jobs to computing sites which have $\mathrm{CE}$ for execution. Computing sites request some resources for running job that they can be files. If these files are not available locally then they will be accessed remotely.

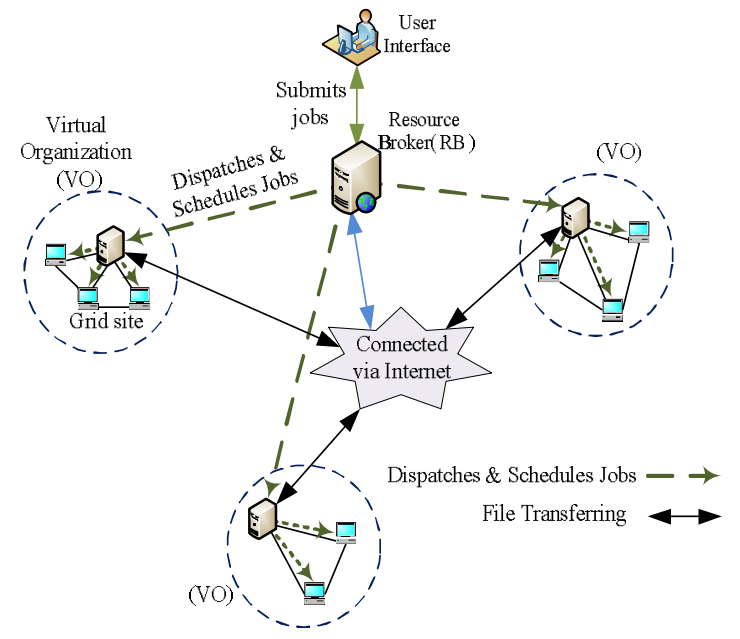

Figure 3. group replication architecture

\subsection{The architecture of LS and grid sites}

In this section, the LS components and components of grid sites and communications between them are presented. Figure
4 shows messages passing between LS components and grid sites.

\subsubsection{LS Components}

LS is composed of two components as follows:

\subsubsection{Replica Catalogue (RC)}

this component maps between physical address and logical name of a replica.

\subsubsection{Replica Manager (RM)}

after getting information from the Replica Catalogue, the RM checks the locations of the physical file and selects the best replica of file. It is important that the file must be fetched from a low cost grid site. OptorSim finds the best replica according to minimum cost of replication and then RM selects replicas that have lowest instance of CE.

\subsubsection{Components of grid sites}

Each grid site is composed of two components as follows:

\subsubsection{Management of Popular Files Group component (MPFG)}

This component is composed of two parts as follows:

- Access history database: Each job must have access to a set of requested files and send them to a grid site. Sequence of the requested files in each grid site is stored in a local database.

- Produce engine: This component is responsible for processing on database and finding the most popular files group (PFG).

\subsubsection{Group Replication Management component (GRM) \\ In a predefined time interval, this component sends a request for MPFG component. Replacement Management (RM) is part of Replication Management. If there is not enough space for replicating of the PFG to a requester grid site, then RM will replace the PFG with old replica according to the replacement algorithm.}

\subsection{Communication between grid sites and LS}

Internal components of proposed architecture and messages passing between them are shown in figure 3 . It consists of two new components which are shown by dashed lines in figure 3 . At the beginning of time interval GRM sends a replication request to MPFG component. MPFG processes on the access history database and finds PFG by using existing replication algorithm. Then it sends the requested replica of PFG to LS of grid sites. Also, it sends the name of requester site to LS with each replication request. Then, Replica Catalogue component finds physical addresses of each replica of PFG and transfers them to the Replica Manager component. RM checks the locations of the physical files and determines the best replica by minimum cost of replication. Finally, it will begin the group replication process for the requester sites. 


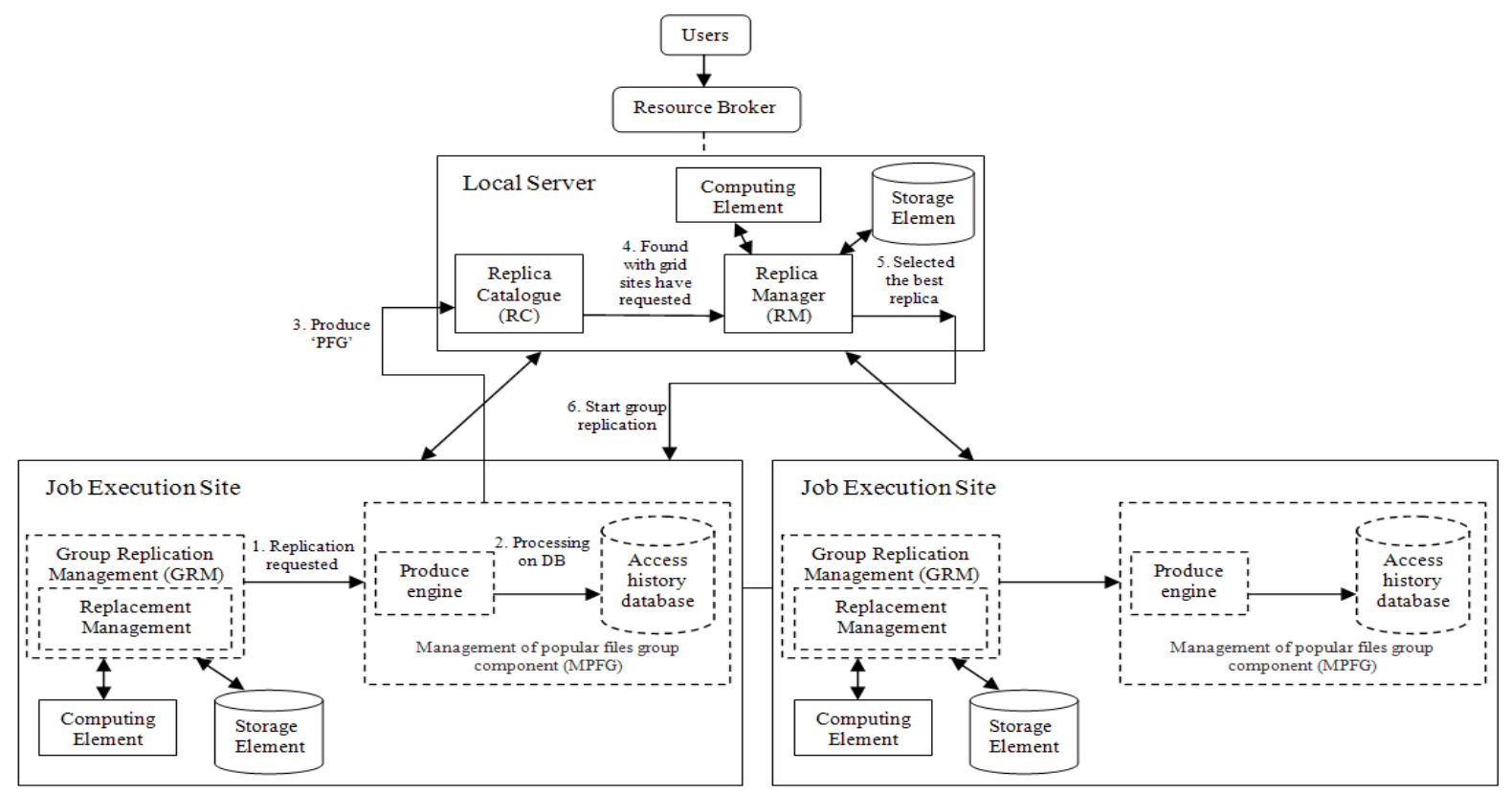

Figure 4. Messages passing between grid sites and LS.

\section{CONCLUSION AND FUTURE WORK}

In grid systems, heterogeneous computational resources and data should be shared between independent organizations that are scatter geographically. On the other hand, the data Grid is a kind of grid services that provides services and infrastructure for distributed data applications with large volumes of data. Data replication is one of the main services for managing volume data in data grid. This service saves replica in different locations. Grid architecture has an efficient factor in replication technique. In this paper, we proposed a new architecture for group replication in data grid. We added two components in our architecture, (MPFG) for Management of Popular Files Group and (GRM) for Group Replication Management. By using this architecture, popular files group will be replicated in grid sites at the end of predefined time interval. For future works, we plan to implement this architecture and to combine it with group replication and replacement technique.

\section{REFERENCES}

[1] Foster, I., Kesselman, C., Tuecke, S., "The anatomy of the grid: Enabling scalable virtual organizations", International Journal of Supercomputer Applications, pp. 200-222, 2001.

[2] Jacob, B., Brown, M., Fukui, K., Trivedi, N., "Introduction to Grid Computing ",IBM Red books , pp. 3-6, 2005.

[3] Amjad, T., Sher, M., Daud, A., "A survey of dynamic replication strategies for improving data availability in data grids", Future Generation Computer Systems 28, pp.337-349, 2012.

[4] Ming-Chang, L., Fang-Yie, L., Ying-ping, Ch., "PFRF: An adaptive data replication algorithm based on star- topology data grids", Future Generation Computer Systems 28, pp.1045-1057, 2012.

[5] The MONARC project. http://monarc.web.cern.ch/MONARC/, [Accessed 15 Jan 2012].

[6] Chang, R-S., Chang, J-S., Lin, S-Y., "Job scheduling and data replication on data grids", Future Generation Computer Systems 23, pp. 846-860, 2007.

[7] Ranganathan, K., Foster, I., "Design and evaluation of dynamic replication strategies for a high performance data grid", in: International Conference on Computing in High Energy an Nuclear Physics, vol. 2001, 2001.

[8] Ranganathan, K., Iamnitchi, A., Foster, I., Improving data availability through dynamic model driven replication in large peer-to-peer communities, in: CCGrid, p. 376, 2002.

[9] Chang, R-S., Chang, H-P., Wang, Y-T., "A Dynamic Weighted Data Replication Stratrgy in Data Grids", The Journal of Supercomputing, 45(3), pp. 277-295, 2008.

[10] Park, S.-M., Kim, J.-H., Go, Y.-B., Yoon, W.-S., "Dynamic grid replication strategy based on internet hierarchy, in: International Workshop on Grid and Cooperative Computing", in: Lecture Note in Computer Science, vol. 1001, pp. 1324-1331, 2003.

[11] Sepahvand, R., Horri, A., Dastghaibyfard, Gh., "A New 3-layer Replication and Scheduling Strategy in Data Grid", International Symposium on Telecommunications, pp. 464-469, 2008.

[12] Sashi, K., Thanamani, A.S., "Dynamic replication in a data grid using a Modified BHR Region Based Algorithm", Future Generation Computer Systems 27, pp. 202-210, 2011

[13] Saadat, N., rahmani, A-M., "PDDRA: A new prefetching based dynamic data replication algorithm in 
International Journal of Computer Applications Technology and Research

Volume 2- Issue 2, 147 - 151, 2013, ISSN: 2319-8656

data grids", Future Generation Computer Systems 28, pp. 666-681, 2012.

[14] Tian, T., Luo, J., Wu, Z., Song, A., "A pre-fetchingbased replication algorithm in data grid, in: 3th International Conference on Pervasive Computing and Applications", pp. 526-531, 2008.

[15] The European DataGrid Project, http://www.edg.org, [Accessed 15 Jan 2012].

[16] Bell, W.H., Cameron, D.G., Capozza, L., Millar, A.P., Stockinger, K., Zini, F., "Simulation of dynamic grid replication strategies in OptorSim", International Journal of High performance Computing Applications 17 (4), 2003.
[17] CMS Data Challenge, 2004: http://www.uscms.org/s\&c/dc04/, [Accessed 15 Jan 2012].

[18] Cameron, D.G., Millar, A.P., Nicholson, C., “OptorSim: a simulation tool for scheduling and replica optimization in data grids", Proceedings of Computing in High Energy and Nuclear Physics (CHEP), 2004.

[19] OptorSim - A Replica Optimiser Simulation, http://griddata-management.web.cern.ch/grid-data-

management/optimization/optor, [Accessed 15 Jan 2012]. 